
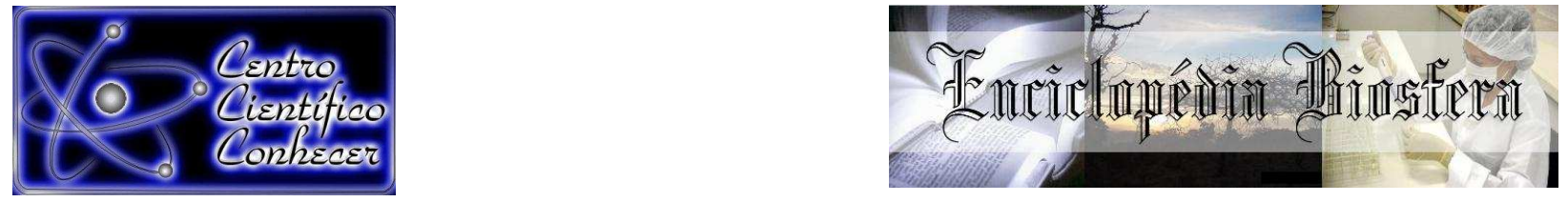

\title{
PROSPECÇÃO DE ARTIGOS CIENTÍFICOS PUBLICADOS SOBRE QUALIDADE DE ENSINO NA EDUCAÇÃO SUPERIOR
}

\author{
Laís Simonelli ${ }^{1}$, Ehimar da Silva Júnior ${ }^{2}$, Cássio Cecato Favarato ${ }^{3}$, George Simonelli ${ }^{4}$ \\ ${ }^{1}$ Arquiteta e Urbanista, Faculdades Integradas de Aracruz. Pós-graduada em \\ Docência do Ensino Superior, (laissimonelli@hotmail.com). Espírito Santo - Brasil. \\ ${ }^{2}$ Graduando em Engenharia Elétrica, Universidade Salvador. \\ ${ }^{3}$ Mestre em Física, Universidade Federal do Espírito Santo \\ ${ }^{4}$ Professor Assistente da Universidade Federal da Bahia.
}

Recebido em: 03/10/2016 - Aprovado em: 21/11/2016 - Publicado em: 05/12/2016 DOI: 10.18677/EnciBio_2016B_145

\begin{abstract}
RESUMO
O progresso tecnológico tem exigido nas mais diferentes áreas a necessidade de mão-de-obra qualificada. Para ajudar a atender a essa demanda destacam-se as instituições de ensino superior. Para cumprir adequadamente seu papel perante a sociedade, essas instituições precisam prezar pela excelência na qualidade de ensino. Esta pesquisa objetivou a realização de uma prospecção de artigos publicados na plataforma ScienceDirect nos últimos dez anos sobre educação superior de qualidade. Os resultados mostraram que o número de trabalhos publicados nessa temática tem crescido, e que os artigos mais recentes têm tratado do uso de novas tecnologias na adoção de métodos de avaliação da qualidade do ensino.
\end{abstract}

PALAVRAS-CHAVE: Ensino superior, Prospecção, Qualidade.

\section{PROSPECT OF PAPERS PUBLISHED ON EDUCATION QUALITY IN HIGHER EDUCATION}

\begin{abstract}
Technological progress is required in different areas the need for skilled labor. To help meet this demand there are the higher education institutions. To properly fulfill their role in society, these institutions need to appreciate the excellence in the quality of education. This research aimed to conduct a survey of articles published in ScienceDirect platform over the past decade on quality higher education. The results showed that the number of papers published in this issue has grown, and that the most recent articles have dealt with the use of new technologies in the adoption of methods of assessing the quality of education.
\end{abstract}

KEYWORDS: Higher Education, Quality, Prospection.

\section{INTRODUÇÃO}

A formação de profissionais para atuar em diferentes áreas é essencial para a sociedade. Para que os mesmos possam atender satisfatoriamente as demandas sociais é necessário o acesso a educação de qualidade, seja em nível técnico ou superior. Para os propósitos do aprendizado serem alcançados é importante 
determinar alguns critérios, tais como: perspectivas da coletividade, os anseios dos alunos, o perfil dos profissionais procurados pelas companhias, bem como as carências das entidades competentes (BERGSMANN et al., 2015).

Não há na literatura uma definição unânime para qualidade. Isso ocorre porque o termo pode ser empregado em campos variados e com diferentes sentidos. No caso da área de educação, a ideia de qualidade deve ser estabelecida de acordo com certos valores, propósitos e imposições da sociedade em determinado período e local (NETTO et al., 2010). Segundo BENNETT et al. (2015), o significado de qualidade poderia estar relacionado à identificação de metas para aprendizagem, utilização de métodos e condições para que os discentes possam atingir objetivos previamente definidos.

Analisar a qualidade da educação não é uma temática recente. Frente aos estímulos da universalização do ensino, a qualidade surge como elemento decisivo na formação dos profissionais (NETTO et al., 2010). Para MIGUEL et al. (2001) e ANDRÉ et al. (2016), as universidades e faculdades, como entidades responsáveis pela formação das pessoas que serão encarregadas pela evolução cultural, técnica e científica de um determinado lugar, podem ser consideradas meios que necessitam de constante avaliação de qualidade.

No Brasil, com o aumento do acesso a educação de nível superior torna-se cada vez mais importante a investigação da qualidade dos cursos ofertados. Um dos motivos que afetam a qualidade da educação superior é o crescimento exagerado das instituições privadas (NETTO et al., 2010).

SPOOREN et al. (2014) afirmam que para melhorar a qualidade do ensino superior os docentes devem variar os métodos utilizados no ensino, adequando-se aos discentes e distinguindo as particularidades de cada um, colaborando desta forma, para tornar mais satisfatório o processo de ensino e de aprendizagem no ambiente escolar. Tendo em vista o cenário descrito, esta pesquisa consistiu na realização de uma prospecção de artigos científicos publicados nos últimos dez anos sobre qualidade de ensino na educação superior. Assim, foi possível quantificar os artigos e apontar o que tem sido investigado recentemente na temática de interesse.

\section{MATERIAL E MÉTODOS}

Para atingir o objetivo deste trabalho foi necessário pesquisar artigos científicos na base de dados da plataforma online do ScienceDirect. O ScienceDirect possui cerca de 14 milhões de publicações, 3800 periódicos e mais de 35000 livros. Podem ser encontrados trabalhos nas áreas de ciências exatas, biológicas, sociais, humanas e da saúde.

As palavras-chave utilizadas na prospecção foram Higher education, Teaching practices e Quality education. Foram realizadas três buscas distintas. Na primeira busca utilizou-se o termo Educação superior (Higher education). Na segunda busca juntou-se os termos Higher education e Práticas de ensino (Teaching practices). O termo de junção empregado entre as palavras-chave foi "and". Na última busca juntou-se os termos Higher education, Teaching practices e Educação de qualidade (Quality education). As palavras escolhidas para a busca foram pesquisadas em todos os periódicos (Journals - All Sciences) no resumo, título e palavras-chave (Abstract, Title, Keywords). Optou-se por artigos de acesso livre, e artigos de acesso por assinatura. A pesquisa foi realizada em Advanced search, no ambiente apresentado na Figura 1. 


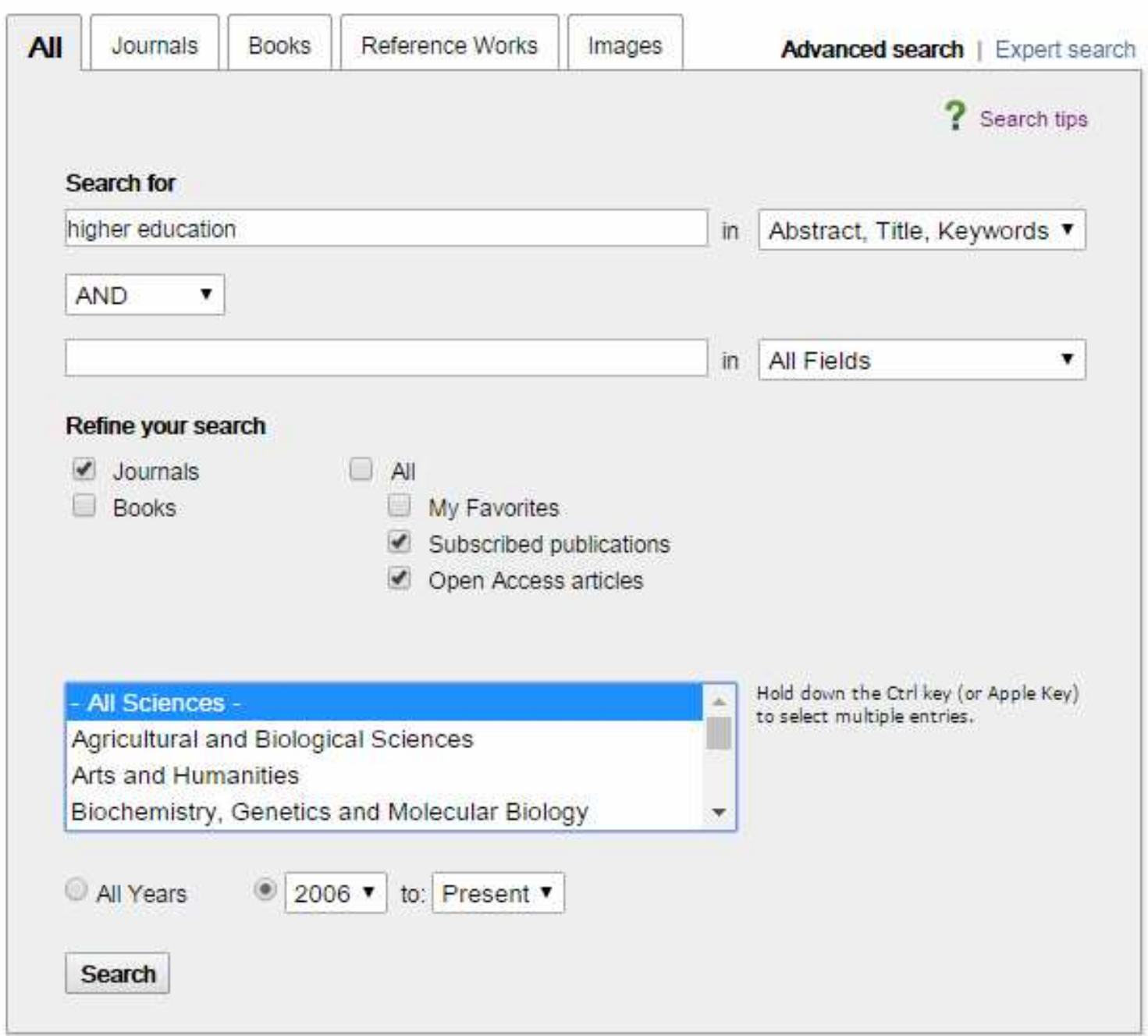

FIGURA 1 - Ambiente de pesquisa avançada da plataforma SciencDirect. Fonte: SCIENCEDIRECT (2016).

Para cada uma das três pesquisas registrou-se o número total de publicações encontradas, e a quantidade de trabalhos publicados por ano nos últimos dez anos (2006 até a presente data). Os resultados da pesquisa foram organizados em quadros e gráficos, e os trabalhos internacionais mais recentes encontrados com as palavras-chave Higher education, Teaching practices e Quality education destacados.

\section{RESULTADOS E DISCUSSÃO}

Utilizando-se a metodologia definida foi possível obter o número de trabalhos publicados para as diferentes palavras-chave utilizadas na pesquisa, conforme mostra o Quadro 1.

QUADRO 1 - Número de trabalhos publicados

\begin{tabular}{cccc}
$\begin{array}{c}\text { Higher } \\
\text { education }\end{array}$ & $\begin{array}{c}\text { Teaching } \\
\text { practices }\end{array}$ & $\begin{array}{c}\text { Quality } \\
\text { education }\end{array}$ & $\begin{array}{c}\text { Número de } \\
\text { publicações }\end{array}$ \\
\hline X & & & 23122 \\
X & $X$ & & 834 \\
X & $X$ & $X$ & 178 \\
\hline
\end{tabular}


De acordo com o Quadro 1, a medida que se aumenta o número de palavraschave menos artigos são encontrados. Foram encontradas 178 publicações para a utilização conjunta dos termos Higher education, Teaching practices e Quality education. As Figuras 2, 3 e 4 mostram a evolução do número de artigos publicados de 2012 a 2016.

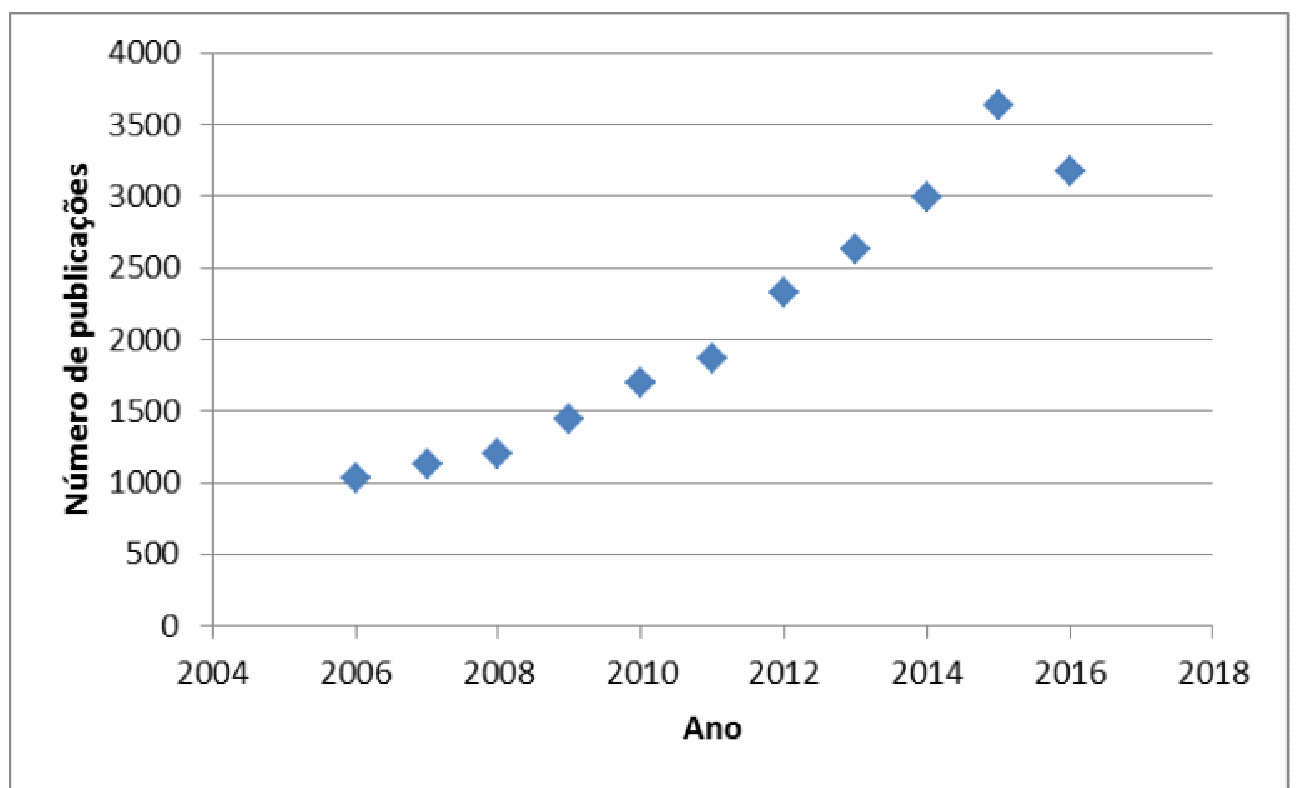

FIGURA 2 - Trabalhos publicados por ano. Palavra-chave: Higher education.

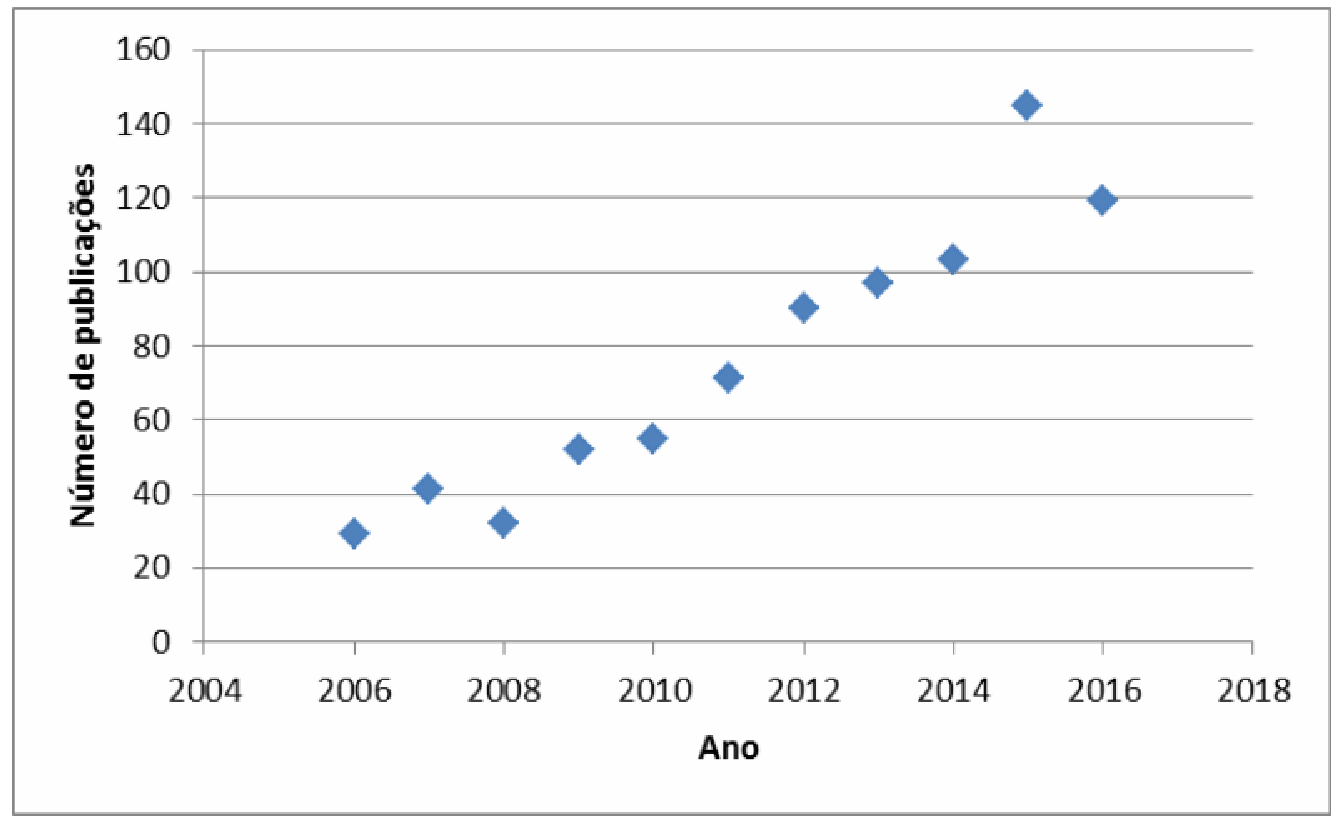

FIGURA 3 - Trabalhos publicados por ano. Palavras-chave: Higher education e Teaching practices. 


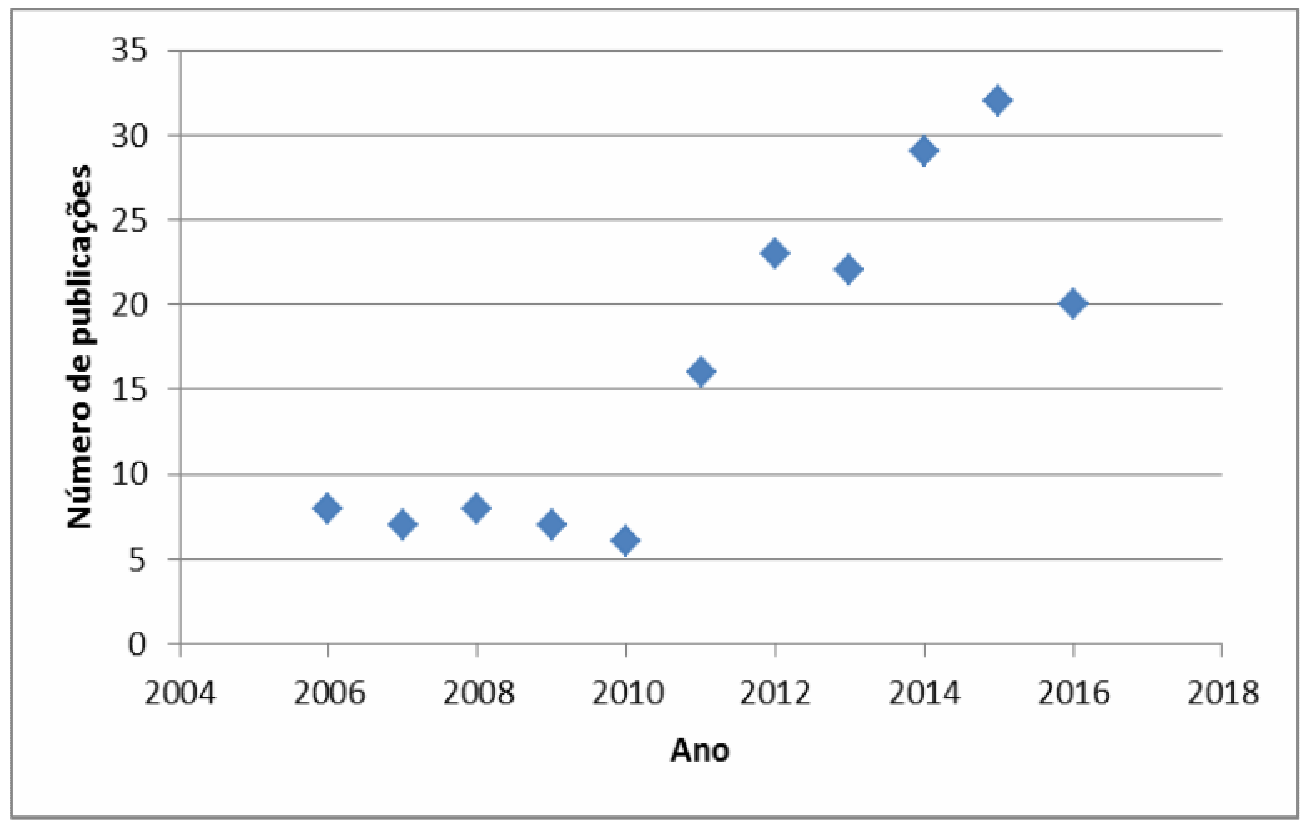

FIGURA 4 - Trabalhos publicados por ano. Palavras-chave: Higher education, Teaching practices e Quality education.

Conforme mostram as Figuras 2, 3 e 4 há uma tendência geral no aumento do número de publicações no período de 2006 a 2016. Isso mostra o interesse crescente dos pesquisadores da área da educação pelo ensino de nível superior. Para que as instituições de ensino superior cumpram seu papel é fundamental o avanço constante na qualidade de ensino.

Para verificar a qualidade do ensino superior de uma instituição é comum a realização de avaliações. Geralmente utilizam-se questionários que são preenchidos por alunos, e até mesmo professores. Esse tipo de instrumento de avaliação na maioria dos casos é limitado, pois dificilmente conseguirá avaliar se as competências necessárias foram desenvolvidas pelo discente.

BERGSMANN et al. (2015) relatam sobre a necessidade de um conceito de avaliação mais abrangente, e que contribua para a melhoria da verificação das competências aprendidas pelos alunos em instituições de ensino superior. Os autores propõem que a avaliação ocorra de modo participativo, e em três etapas. $\mathrm{Na}$ primeira etapa deve-se avaliar se os alunos possuem condições de desenvolver as competências necessárias para a sua formação através do currículo definido para o curso. Na segunda etapa deve-se verificar se os estudantes estão realmente adquirindo as competências importantes. Por fim, na terceira etapa deve-se avaliar aspectos concretos do processo de ensino, como por exemplo, a metodologia usada pelos professores. Segundo estes autores as instituições de ensino superior devem promover avaliações internas buscando desenvolver e acompanhar os currículos. Os resultados obtidos por ANDRÉ et al. (2016) corroboram com a pesquisa de BERGSMANN et al. (2015).

SPOOREN et al. (2014) afirmam que os questionários preenchidos pelos alunos no ensino superior são valiosos instrumentos de diagnóstico e acompanhamento da qualidade de ensino. Os autores recomendam a elaboração de questionários curtos e utilização de análise fatorial na interpretação dos resultados.

SPOOREN \& VAN LOON (2012) destacam que a avaliação da qualidade do ensino através de questionários online se tornou uma prática muito comum nas universidades. Apesar dos questionários online serem bastante práticos, os autores 
relatam que a quantidade de alunos que os respondem é geralmente baixa, prejudicando a representatividade da amostra. Os autores usam modelo logístico multinível para analisar os impactos estatísticos que os questionários não respondidos provocam.

Em um estudo realizado na Índia por SINGH \& SARKAR (2015) foi observado que a baixa qualidade do ensino público no país sobrecarrega as escolas privadas. Segundo os autores os alunos de cálculo das escolas particulares apresentam pontuação significativamente maior que as obtidas por alunos de escolas públicas. Foi relatado no estudo que a qualificação profissional do professor, a distância percorrida pelo docente até o trabalho, os livros utilizados e a relação professor aluno são aspectos determinantes na qualidade do ensino.

Segundo JAGGARS \& XU (2016) outro aspecto que afeta a qualidade da educação superior é a modalidade do curso. Os cursos de graduação realizados a distância com aulas online são muito úteis, porém, há pouco consenso sobre a maneira de estruturá-los de modo a garantir a aprendizagem de qualidade. Os autores afirmam que a relação interpessoal geralmente impacta positivamente a aprendizagem do aluno, e, portanto, é recomendável que haja interação frequente entre professores e alunos nos ambientes virtuais de aprendizagem dos cursos online. Isso é capaz de despertar nos alunos mais comprometimento e perseverança.

BENNETT et al. (2015) afirmam que a tecnologia é uma ferramenta útil para dinamizar e melhorar a qualidade do ensino. As experiências de aprendizagem online são importantes para ampliar o contato dos alunos com os conteúdos. Os autores afirmam que os professores devem usar a tecnologia de modo planejado e com objetivos bem definidos. Nesse contexto, DLOUHÁ \& BURANDT (2015) defendem a transdisciplinaridade como meio necessário para alcançar a qualidade no ensino.

Um estudo realizado no Quênia por NGWARE et al. (2014) mostrou que o estilo de ensino do professor desempenha papel importante nas diferenças de desempenho acadêmico encontradas. Assim sendo, a qualidade do aprendizado está relacionada com a prática docente utilizada, que pode explicar as diferenças de desempenho entre alunos e escolas. A pesquisa de AITYSHEVA \& JUMAGELDINOV (2014) possuem resultados que corroboram nesse sentido.

\section{CONCLUSÕES}

Com a prospecção realizada no ScienceDirect foi possível alcançar o objetivo da pesquisa. Os resultados obtidos são relevantes, uma vez que trabalhos com esta abordagem são escassos. De acordo com o observado no estudo, recomenda-se que investigações futuras em educação superior levem em consideração o uso de novas tecnologias nas universidades, especialmente na avaliação da qualidade do ensino.

\section{REFERÊNCIAS}

AITYSHEVA, A.; JUMAGELDINOV, A. The Use of the Methods of Psychological Consultation in the Education Process. Procedia-Social and Behavioral Sciences, v. 114, p. 749-753, 2014.

ANDRÉ, B.; AUNE, A. G.; BRAEND, J. A. Embedding evidence-based practice among nursing undergraduates: Results from a pilot study. Nurse education in practice, v. 18, p. 30-35, 2016. 
BENNETT, S.; AGOSTINHO, S.; LOCKYER, L. Technology tools to support learning design: Implications derived from an investigation of university teachers' design practices. Computers \& Education, v. 81, p. 211-220, 2015.

BERGSMANN, E., SCHULTES, M. T., WINTER, P., SCHOBER, B., SPIEL, C. Evaluation of competence-based teaching in higher education: From theory to practice. Evaluation and program planning, v. 52, p. 1-9, 2015.

DLOUHÁ, J.; BURANDT, S. Design and evaluation of learning processes in an international sustainability oriented study programme. In search of a new educational quality and assessment method. Journal of Cleaner Production, v. 106, p. 247258, 2015.

JAGGARS, S. S.; XU, D. How do online course design features influence student performance?. Computers \& Education, v. 95, p. 270-284, 2016.

MIGUEL, J. M.; CAIS, J.; VAQUERA, E. Excelência: calidad de las universidades españolas. Madrid: EFCA, 2001.

NETTO, C.; GIRAFFA, L.; FARIA, E. Graduações a distância e o desafio da qualidade. Porto Alegre: EDIPUCRS, 2010.

NGWARE, M. W.; OKETCH, M.; MUTISYA, M. Does teaching style explain differences in learner achievement in low and high performing schools in Kenya?. International Journal of Educational Development, v. 36, p. 3-12, 2014.

SINGH, R.; SARKAR, S. Does teaching quality matter? Students learning outcome related to teaching quality in public and private primary schools in India. International Journal of Educational Development, v. 41, p. 153-163, 2015.

SPOOREN, P.; MORTELMANS, D.; CHRISTIAENS, W. Assessing the validity and reliability of a quick scan for student's evaluation of teaching. Results from confirmatory factor analysis and G Theory. Studies in Educational Evaluation, v. 43, p. 88-94, 2014.

SPOORENA, P.; VAN LOON, F. Who participates (not)? A non-response analysis on students' evaluations of teaching. Procedia-Social and Behavioral Sciences, v. 69, p. 990-996, 2012. 